\title{
Phase behaviour of three-component lipid mixtures
}

\author{
S Komura ${ }^{1,3}$, H Shirotori $^{1}$ and P D Olmsted ${ }^{2}$ \\ ${ }^{1}$ Department of Chemistry, Faculty of Science, Tokyo Metropolitan University, Tokyo 192-0397, \\ Japan \\ ${ }^{2}$ School of Physics and Astronomy, University of Leeds, Leeds LS2 9JT, UK \\ E-mail: komura@comp.metro-u.ac.jp
}

Received 30 December 2004, in final form 25 January 2005

Published 22 July 2005

Online at stacks.iop.org/JPhysCM/17/S2951

\begin{abstract}
To describe the lateral phase separation in biological membranes, we propose phenomenological models for binary and ternary lipid mixtures. We consider a coupling between the local lipid composition and internal membrane structure, and its influence on transitions between liquid and gel membrane phases. Assuming that the gel transition temperature of a pure lipid is shifted by the presence of other lipids, we obtained a variety of binary phase diagrams which are in semi-quantitative agreement with the experiments. With the use of the model parameters deduced from the binary cases, we predict the quantitative phase behaviour of the ternary lipid systems.
\end{abstract}

\section{Introduction}

Biological membranes are lipid bilayers in water, and typically contain various components such as lipid mixtures, sterols, and proteins that are indispensable to cell functions [1]. Rather than being uniformly distributed in the membrane, there is growing evidence that some membrane components are incorporated in domains arising from lateral lipid segregation in membranes. This phenomenon has attracted great interest in the context of 'rafts' [2], i.e., liquid domains rich in cholesterol, saturated lipids (typically sphingomyelin lipids), and in some cases particular proteins [3]. Moreover, cholesterol-rich domains have been directly observed in model membranes composed of lipid mixtures and cholesterol, using fluorescence microscopy [4-7].

From a physical viewpoint, a strategy for understanding the basic structure of 'rafts' in biological membranes is as follows [8]. First, it is necessary to have a simple model for binary mixtures of saturated and unsaturated lipids. Second, a minimal model describing binary lipidcholesterol systems is required in order to understand the effects of cholesterol on membrane

3 Author to whom any correspondence should be addressed. 
phase behaviour. Finally, these two viewpoints may be combined to fully investigate threecomponent systems. In the previous paper, we have focused on the first two steps and proposed simple phenomenological models for lipid-lipid and lipid-cholesterol binary mixtures [9]. Assuming that the gel transition temperature of the saturated lipid is shifted by the presence of the unsaturated lipid, and that cholesterol acts as an external field on the chain melting transition, we obtained a variety of phase diagrams which are in semi-quantitative agreement with the experiments. Such an approach proved to be quite useful since we can predict, at least qualitatively, the complex phase behaviour of the ternary lipid-lipid-cholesterol system from the three binary sub-systems. However, a complete model which quantitatively describes the ternary phase behaviour is still lacking, although there are several experimental attempts to determine it [10-13].

In this paper, we propose a model for three-component phospholipid mixtures (without cholesterol). This model is a natural extension of our previous model for binary lipid mixtures [9], and provides us with the first step to construct a general ternary model including sterols. Our model can be useful, since red blood cell membranes, for example, contain over 250 lipid species [14]. In our approach, we first determine the phenomenological parameters from the three experimental phase diagrams of binary systems. These values are used to predict the full ternary phase behaviour based on the extended model for three-component lipid systems. Although there have been many experimental works on binary lipid mixtures, we are not aware of any corresponding systematic study for ternary lipid systems. Hence our theoretical predictions should be checked in the future. In the next section, we first describe the model for binary phospholipid systems. Our previous model is slightly modified in order to take into account the asymmetry between the two lipids. The calculated phase diagrams reproduce experimental ones, without specifying the detailed microscopic state of the lipids. In section 3, the model for three-component phospholipid mixtures is presented together with some typical ternary phase diagrams.

\section{Binary lipid systems}

Lipid membranes undergo a freezing or gel transition, in which the hydrocarbon tails order. It is generally believed that the gel phase transition is driven by the significant reduction in lateral diffusion as well as conformational ordering of lipids. However, because these two degrees of freedom are strongly coupled, the gel phase transition occurs at a single temperature for pure lipid systems. As explained below, one of our major assumptions is that the membrane state, even for lipid mixtures, can be described by one internal degree of freedom, coupled to the lateral phase separation.

Let us first focus on a binary lipid system for which many experiments have been performed [15]. If different lipids exhibit only a small difference in their gel transition temperature, their composition-temperature phase diagram will include a 'cigar-like' shape of liquid-gel coexistence (figure 1(a)). Below this liquid-gel coexistence, there is a gelgel coexistence region (figure 1(b)) unless the water freezes around this temperature. When the gel transition temperature difference between the two lipids becomes larger, the phase diagram becomes more complex because the two coexistence regions partially overlap (figure 1(c)).

We consider a single bilayer membrane composed of $x$ mole fraction of lipid A and $y=1-x$ of different lipid B. The two lipids are taken to have different gel transition temperatures originating from different chain length, degree of saturation, or hydrophilic head group. The total free energy $f=f_{1}+f_{2}$ comprises (i) the free energy $f_{1}$ of mixing and binary interactions, and (ii) the 'stretching' energy $f_{2}$, which controls changes in bilayer thickness. 

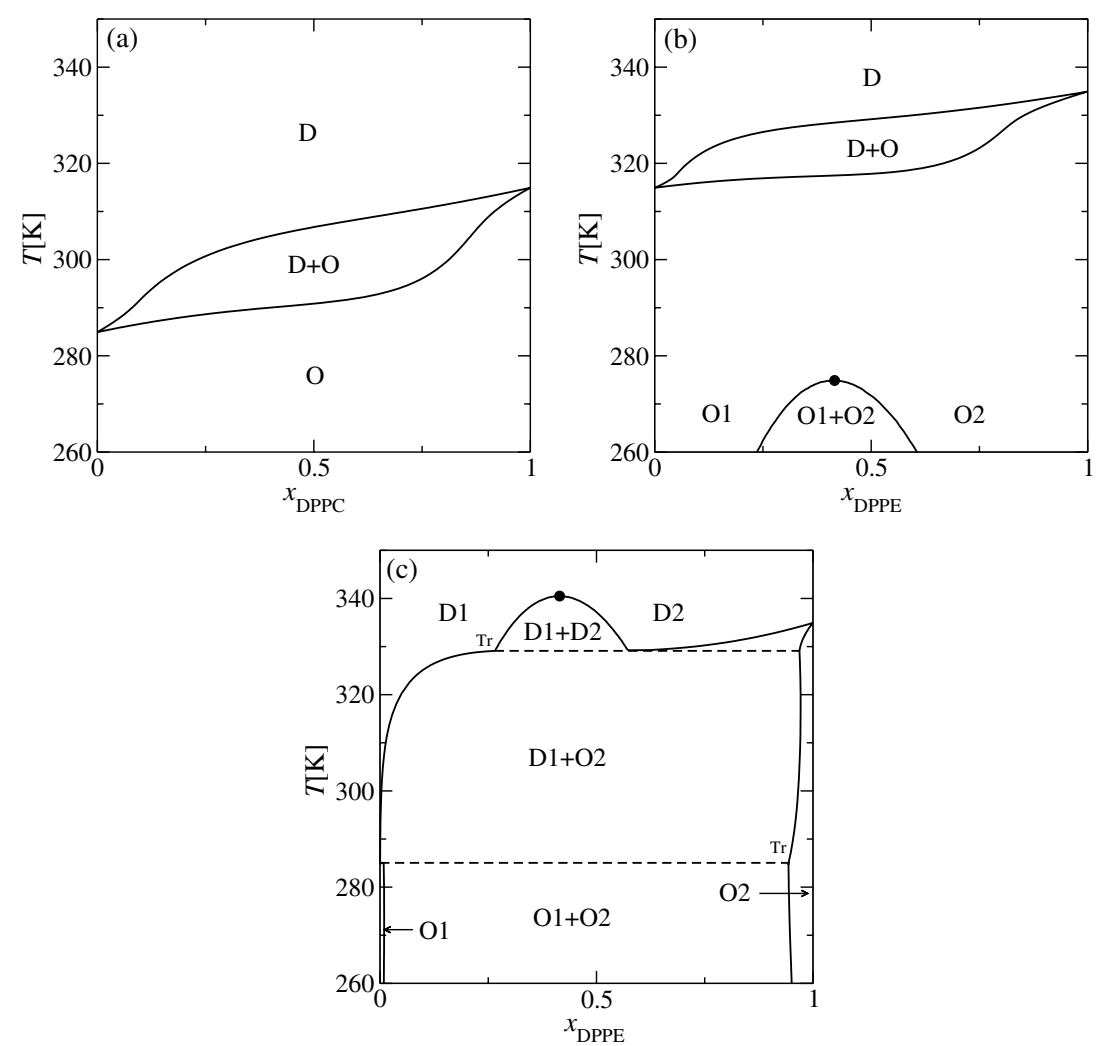

Figure 1. Calculated phase diagrams of binary lipid mixtures corresponding to (a) DEPC-DPPC, (b) DPPC-DPPE and (c) DEPC-DPPE. The interaction parameters are (a) $J=1.0 \times 10^{-20} \mathrm{~J}$, (b) $J=1.0 \times 10^{-20} \mathrm{~J}$, (c) $J=1.37 \times 10^{-20} \mathrm{~J}$. The parameters used for each lipid are $n_{\mathrm{DEPC}}=1, n_{\mathrm{DPPC}}=1, n_{\mathrm{DPPE}}=2, T_{\mathrm{DEPC}}^{*}=234 \mathrm{~K}, T_{\mathrm{DPPC}}^{*}=264 \mathrm{~K}, T_{\mathrm{DPPE}}^{*}=284 \mathrm{~K} . \mathrm{The}$ other common parameters are $a_{2}^{\prime}=2.4 \times 10^{-21} \mathrm{~J} \mathrm{~K}^{-1}, a_{3}=-1.1 \times 10^{-18} \mathrm{~J}, a_{4}=2.2 \times 10^{-18} \mathrm{~J}$, $\delta_{0}=3.5 \times 10^{-9} \mathrm{~m}$. The ordered (large $\left.\psi\right)$ and disordered $(\psi=0)$ phases are respectively denoted by $\mathrm{O}$ and $\mathrm{D}$. The critical point is indicated by a filled circle, and the triple point by Tr.

The free energy per site $f_{1}$ is the sum of the entropy of mixing and enthalpy. It can be written within a Flory-Huggins (mean-field) theory as

$$
f_{1}(x)=k_{\mathrm{B}} T\left[\frac{x}{n_{\mathrm{A}}} \log x+\frac{1-x}{n_{\mathrm{B}}} \log (1-x)\right]+\frac{1}{2} J x(1-x),
$$

where $k_{\mathrm{B}}$ is the Boltzmann constant, $T$ is the temperature, and $J>0$ is an attractive interaction parameter that enhances demixing. The overall asymmetry between the two lipids that contributes to the entropy is taken into account through $n_{\mathrm{A}}$ and $n_{\mathrm{B}}$. These phenomenological parameters will be adjusted so as to reproduce the location of the experimentally determined critical point.

To describe the gel transition, which involves chain ordering and stiffening, we introduce a rescaled membrane thickness $\psi \equiv\left(\delta-\delta_{0}\right) / \delta_{0}$ as an order parameter, where $\delta$ is the actual membrane thickness, and $\delta_{0}$ is the constant membrane thickness in the disordered phase corresponding to the liquid phase [16]. Note that $\psi$ summarizes changes in various degrees of freedom, including the conformations of the hydrocarbon chains and their interchain correlations. Since the gel transition is first order, an appropriate phenomenological 
Landau expansion of the 'stretching' free energy in powers of $\psi$ is [16]

$$
f_{2}(x, \psi)=\frac{1}{2} a_{2}^{\prime}\left[T-T^{*}(x)\right] \psi^{2}+\frac{1}{3} a_{3} \psi^{3}+\frac{1}{4} a_{4} \psi^{4}
$$

where $a_{2}^{\prime}>0, a_{3}<0$, and $a_{4}>0$ are the phenomenological coefficients which are quantitatively determined according to the experiments. For a single component membrane (i.e., $x=0$ or 1 ), $T^{*}$ is a reference temperature, and the first-order gel transition temperature is given by $T_{\mathrm{g}}=T^{*}+2 a_{3}^{2} /\left(9 a_{2}^{\prime} a_{4}\right)$. For a binary mixture, on the other hand, we describe the reference temperature $T^{*}(x)$ as a linear interpolation between the two pure limits:

$$
T^{*}(x)=x T_{\mathrm{A}}^{*}+(1-x) T_{\mathrm{B}}^{*},
$$

where $T_{\mathrm{A}}^{*}$ and $T_{\mathrm{B}}^{*}$ are the reference temperatures of the pure A and B lipids, respectively. Note that equation (3) leads to a coupling term $x \psi^{2}$. We have neglected a lower order bilinear term $x \psi$, which induces a small temperature and composition dependence in $\delta_{0}$. Another possible coupling term $x^{2} \psi$ simply renormalizes the interaction parameter $J$.

Combining equations (1)-(3), we obtain the total free energy $f$. After minimizing with respect to $\psi$, the two-phase region is obtained by the Maxwell construction. As regards the choice of the parameter values, we tried to reproduce the phase diagrams of the binary lipid mixtures reported in $[17,18]$. In figure 1 , we show three phase diagrams representing (a) DEPC-DPPC, (b) DPPC-DPPE and (c) DEPC-DPPE mixtures. The mixture of DEPCDPPC, as shown in figure 1(a), corresponds to that with relatively weak segregation tendency characterized by small $J$. In this case, a cigar-like coexistence region is obtained between a disordered phase (D) where $\psi=0$ (liquid) and an ordered phase $(\mathrm{O})$ where $\psi>0$ (gel). With the same value of $J$ but with a different combination of transition temperatures, as in figure 1(b), there appears another coexistence region between two ordered phases, $\mathrm{O} 1+\mathrm{O} 2$, below the cigar-shape region. These two ordered phases are characterized by the different order parameter values, $\psi_{1}<\psi_{2}$, which coincide at the critical point.

The phase diagram for DEPC-DPPE mixtures can be reproduced by choosing larger $J$ as shown in figure $1(\mathrm{c})$, in which the $\mathrm{O} 1+\mathrm{O} 2$ coexistence region extends into and beyond the $\mathrm{D}+\mathrm{O}$ coexistence region. We chose here $n_{\mathrm{DEPC}}=1$ and $n_{\mathrm{DPPE}}=2$. It should be noted that the DEPC-DPPE lipid mixtures have a stronger segregation tendency because both head and tail moieties are different. In this more complex phase diagram, there are two triple points (denoted as Tr) at which the two disordered phases and ordered phase (D1 + D2 + O2), or the disordered phase and two ordered phases $(\mathrm{D} 1+\mathrm{O} 1+\mathrm{O} 2)$ coexist.

\section{Ternary lipid systems}

Based on the model for binary lipid systems, we now discuss the phase behaviour of threecomponent lipid membranes. Suppose a bilayer membrane consists of three types of lipids, A, $\mathrm{B}$ and $\mathrm{C}$, whose mole fractions are $x, y$, and $z$, respectively. Note that there is a conservation law: $x+y+z=1$. The simplest and most natural extension of the binary case is to construct a free energy $f=f_{1}+f_{2}$ such that

$$
\begin{aligned}
& f_{1}(x, y, z)=k_{\mathrm{B}} T\left[\frac{x}{n_{\mathrm{A}}} \log x+\frac{y}{n_{\mathrm{B}}} \log y+\frac{z}{n_{\mathrm{C}}} \log z\right]+\frac{J_{\mathrm{AB}}}{2} x y+\frac{J_{\mathrm{BC}}}{2} y z+\frac{J_{\mathrm{CA}}}{2} z x, \\
& f_{2}(x, y, z, \psi)=\frac{1}{2} a_{2}^{\prime}\left[T-T^{*}(x, y, z)\right] \psi^{2}+\frac{1}{3} a_{3} \psi^{3}+\frac{1}{4} a_{4} \psi^{4},
\end{aligned}
$$

and

$$
T^{*}(x, y, z)=x T_{\mathrm{A}}^{*}+y T_{\mathrm{B}}^{*}+z T_{\mathrm{C}}^{*} .
$$

In addition to $T_{\mathrm{A}}^{*}$ and $T_{\mathrm{B}}^{*}$, the reference temperature of the lipid $\mathrm{C}$ is denoted by $T_{\mathrm{C}}^{*}$. The asymmetry of the lipid $\mathrm{C}$ with respect to the other lipids is taken into account by $n_{\mathrm{C}}$ in the 

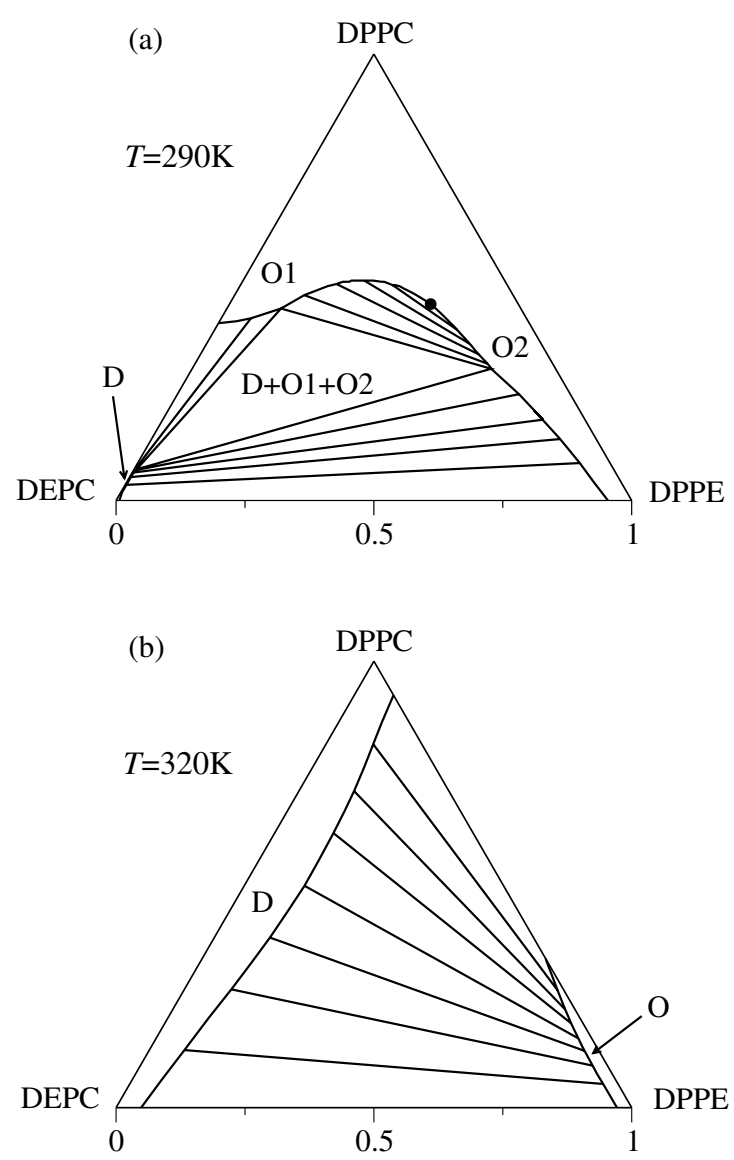

Figure 2. Ternary phase diagrams of the three-component lipid mixture (DEPC-DPPC-DPPE) at (a) $T=290 \mathrm{~K}$ and (b) $320 \mathrm{~K}$. The ordered (large $\psi$ ) and disordered $(\psi=0)$ phases are respectively denoted by $\mathrm{O}$ and $\mathrm{D}$. The solid lines in the two-phase regions represent tie lines. The three-phase coexistence region is denoted by $\mathrm{D}+\mathrm{O} 1+\mathrm{O} 2$. The parameters are the same as those listed in the caption of figure 1 .

entropy terms. Equation (6) brings the couplings terms $x \psi^{2}$ and $y \psi^{2}$. Since the above model naturally reduces to that of the binary systems when one of the species is absent, all the interaction parameters $J_{\mathrm{AB}}, J_{\mathrm{BC}}$, and $J_{\mathrm{CA}}$ can be determined from the corresponding three binary phase diagrams.

To illustrate this procedure, we have investigated the ternary mixture of DEPC-DPPCDPPE. In the previous section, we have obtained three binary phase diagrams which reproduce experimental ones. We use here the same values for the interaction parameters and other phenomenological parameters. For a given composition, the total free energy $f$ is minimized first with respect to $\psi$ to determine the internal state of the membrane. Then the free energies of the two-phase and the three-phase coexisting states are calculated to obtain the whole phase behaviour.

Figure 2 shows two typical phase triangles at (a) $T=290 \mathrm{~K}$ and (b) $320 \mathrm{~K}$. In figure 2(a), the phase separation occurs on the two binary sides (DEPC-DPPE and DEPC-DPPC) of the triangle, and there is a three-phase coexisting region $(\mathrm{D}+\mathrm{O} 1+\mathrm{O} 2)$ within the triangle. 
The two-phase coexisting region between two ordered phases $(\mathrm{O} 1+\mathrm{O} 2)$ extends toward the DPPC-DPPE binary side, and ends at a critical point. Note that the O1- and O2-phases have the same symmetry and are continuously connected. At a higher temperature, as in figure 2(b), the phase separation takes place between the DPPC-DPPE binary system, and the extended two-phase region $(\mathrm{D}+\mathrm{O})$ occupies most of the phase triangle. Such a phase diagram results from the strong segregation tendency of DPPE with respect to both DEPC and DPPC at this temperature.

In summary, we have calculated the phase triangles of the three-component lipid mixture based on the proposed phenomenological model. The model parameters were determined from the experimentally obtained binary phase diagrams.

\section{Acknowledgments}

We thank D Andelman and T Kato for useful discussions. This work is supported by the Japan Society for the Promotion of Science, the Royal Society, the Ministry of Education, Culture, Sports, Science and Technology, Japan under grant No. 15540395.

\section{References}

[1] Lipowsky R and Sackmann E (ed) 1995 Structure and Dynamics of Membranes (Amsterdam: Elsevier)

[2] Simons K and Ikonen E 1997 Nature 387569

[3] Brown D A and London E 2000 J. Biol. Chem. 27517221

[4] Dietrich C, Bagatolli L A, Volovyk Z N, Thompson N L, Levi M, Jacobson K and Gratton E 2001 Biophys. J. 801417

[5] Veatch S L and Keller S L 2002 Phys. Rev. Lett. 89268101

[6] Baumgart T, Hess S T and Webb W W 2003 Nature 425821

[7] Gaus K, Gratton E, Kable E P W, Jones A S, Gelissen I, Kritharides L and Jessup W 2003 Proc. Natl Acad. Sci. 10015554

[8] Lipowsky R and Dimova R 2003 J. Phys.: Condens. Matter 15 S31

[9] Komura S, Shirotori H, Olmsted P D and Andelman D 2004 Europhys. Lett. 67321

[10] Feigenson G W and Buboltz J T 2001 Biophys. J. 802775

[11] de Almeida R F M, Fedorov A and Prieto M 2003 Biophys. J. 852406

[12] Veatch S L and Keller S L 2003 Biophys. J. 853074

[13] Veatch S L, Polozov I V, Gawrisch K and Keller S L 2004 Biophys. J. 862910

[14] Myher J J, Kuksis A and Pind S 1989 Lipids 24396

[15] Sackmann E in reference [1]

[16] Goldstein R E and Leibler S 1989 Phys. Rev. A 401025

[17] Wu S H and McConnell H M 1975 Biochemistry 14847

[18] Arnold K, Lösche A and Gawrisch K 1981 Biochim. Biophys. Acta 645143 